\title{
Influence of non-adiabatic temperature variations on line profile variations of slowly rotating $\beta$ Cep stars and SPBs
}

\section{Non-adiabatic eigenfunctions in the atmosphere of a pulsating star}

\author{
M.-A. Dupret ${ }^{1}$, J. De Ridder ${ }^{2}$, C. Neuforge ${ }^{1}$, C. Aerts ${ }^{2}$, and R. Scuflaire ${ }^{1}$ \\ 1 Institut d'Astrophysique et de Géophysique de l'Université de Liège, avenue de Cointe 5, 4000 Liège, Belgium \\ 2 Institut voor Sterrenkunde, Katholieke Universiteit Leuven, Celestijnenlaan 200 B, 3001 Heverlee, Belgium
}

Received 2 August 2001 / Accepted 31 January 2002

\begin{abstract}
In this study, we compute theoretical line profiles of a non-radially pulsating star, taking the nonadiabatic effects into account. These non-adiabatic effects are especially important in the atmosphere, where the spectral lines are formed, and must be accounted for. In this first paper of the series, we present a new treatment of the perturbed thermal and dynamical equations in the atmosphere of a pulsating star. We apply our formalism to the computation of non-adiabatic eigenfunctions in a typical $\beta$ Cephei star with low order $p$-modes and in a typical slowly pulsating B star with high-order $g$-modes.
\end{abstract}

Key words. stars: oscillations - stars: atmospheres - Line: profiles - stars: variables: $\beta$ Cep stars: variables: slowly fulsating B stars

\section{Introduction}

Pulsating stars offer us a unique opportunity to probe their internal structure and, in turn, refine our knowledge of stellar evolution and test the physics used in the models. Although precise periods can now currently be detected in multi-periodic non-radial pulsators, the identification of the observed modes remains a problem, while it is critical in providing key ingredients for asteroseismic inferences. Different methods of mode identification in pulsating stars have been developed, based on multi-colour photometry (see e.g. Watson 1988; Cugier et al. 1994; Heynderickx et al. 1994; Garrido 2000), or on spectroscopic observations of line-profile variations (Buta \& Smith 1979; Aerts 1996; Telting \& Schrijvers 1997). Up to now, in all the methods based on line-profile variations, the non-adiabatic character of the pulsation was entirely neglected or treated with an ad hoc parameter (Lee et al. 1992; Townsend 1997). The pulsation is always highly non-adiabatic in the superficial layers of a star, i.e. from the transition region (where the thermal relaxation time is of the same order as the pulsation period) to the surface. The use of the adiabatic approximation is thus inappropriate to obtain credible values for eigenfunctions such as $\delta T / T$ or $\delta \boldsymbol{F}$ in the atmosphere.

Send offprint requests to: M.-A. Dupret, e-mail: dupret@astro.ulg.ac.be
Different authors (Dziembowski 1977; Saio \& Cox 1980; Pesnell 1990) have already performed calculations of non-radial non-adiabatic stellar pulsations, but none of their studies includes a detailed treatment of the pulsation in the line forming region. The goal of our study is to derive such a treatment, and to show how the results obtained can be used to obtain more reliable theoretical lineprofile variations. In this paper, we improve the treatment proposed in Dupret (2001), by deriving better equations to model the temperature variations in the atmosphere (Sect. 3.1) and taking more appropriately the radiation into account in the dynamical equations (Sect. 3.2). Our treatment does not use the diffusion approximation in the atmosphere. It is based instead on the hypothesis that the atmosphere remains in radiative equilibrium during the pulsation.

Our approach can be summarized as follows. In order to obtain better eigenfunctions in the atmospheric layers, the stellar model is subdivided into two parts: the interior and the atmosphere. The equations used in the interior are briefly recalled in Sect. 2. As some of them are no longer valid in the atmosphere, we use a more adequate treatment to model the pulsation in that region, as explained in Sect. 3. The eigenfunctions are then computed globally (interior + atmosphere) using the two different sets of differential equations for the two parts of the star, and with the appropriate matching and boundary conditions. We will refer to the layer that connects the two regions 
as the connecting layer. Typically, the depth of the connecting layer corresponds to a Rosseland optical depth $\tau_{\text {ross }}=4$, but our results are not affected by moderate changes in the choice of this layer. The outermost layer of the model corresponds to a very small optical depth $\left(\log \tau_{\text {ross }}=-4.125\right)$. Therefore, the whole line forming region is included in our modeling. In what follows, we use the notation $\delta X$ for the Lagrangian variation of a quantity $X$ and $X^{\prime}$ for its Eulerian variation. For the sake of brevity, we use the notation $\tau$ (resp. $\kappa$ ) for the Rosseland mean optical depth (resp. opacity). As we neglect the influence of rotation on pulsation, for a given spheroidal mode, each scalar quantity is proportional to the spherical harmonic $Y_{\ell}^{m}(\theta, \varphi)$, and the displacement vector is written:

$$
\begin{aligned}
\boldsymbol{\xi}(r, \theta, \varphi)= & \xi_{r}(r) Y_{\ell}^{m}(\theta, \varphi) \boldsymbol{e}_{r}+\xi_{h}(r) \\
& \left(\frac{\partial Y_{\ell}^{m}}{\partial \theta}(\theta, \varphi) \boldsymbol{e}_{\theta}+\frac{1}{\sin \theta} \frac{\partial Y_{\ell}^{m}}{\partial \varphi}(\theta, \varphi) \boldsymbol{e}_{\varphi}\right)
\end{aligned}
$$

where $\xi_{r}(r)$ is the radial part of the displacement in the radial direction and $\xi_{h}(r)$ is the radial part of the displacement in the transversal direction (same notations as in Unno et al. 1989).

\section{Treatment of the stellar interior}

The formalism and numerical method we use to model and compute the non-radial non-adiabatic oscillations in the interior are almost the same as those detailed in Dupret (2001). The only difference is that we adopt the Eulerian perturbation of the gravitational potential instead of the Lagrangian perturbation because it is better suited to the computation of high-order g-modes. We work in the linear approximation, we neglect the influence of rotation on pulsation, we neglect the Lagrangian variation of the convective luminosity (frozen convection) and finally, we use the diffusion approximation to compute the perturbed radiative flux. The equations used in the interior are briefly recalled below. In these equations, $r$ is the radial coordinate, $M_{\mathrm{r}}$ is the mass inside the radius $r, \omega$ is the angular frequency of oscillation, $\psi$ is the gravitational potential, $g$ is the gravity, $P$ is the total pressure, $\rho$ is the density, $S$ is the entropy, $c_{\mathrm{v}}$ is the specific heat at constant volume, $T$ is the temperature, $L$ is the luminosity at radius $r, F_{r}$ is the radial component of the flux, $\ell$ is the degree of the spherical harmonic $Y_{\ell}^{m}(\theta, \varphi), \delta L_{r} \equiv \delta\left(4 \pi r^{2} F_{r}\right)$ and $\epsilon$ is the rate of energy generation (mainly by nuclear reactions). The radial component of the equation of momentum conservation reads:

$\omega^{2} \xi_{r}=\frac{\partial \psi^{\prime}}{\partial r}+\frac{\partial\left(g \xi_{r}\right)}{\partial r}+\frac{\partial(\delta P / P)}{\partial r} \frac{P}{\rho}+\left(\frac{\delta \rho}{\rho}-\frac{\delta P}{P}\right) g$.

The transversal component of the equation of momentum conservation reads:

$\omega^{2} \xi_{h}=\frac{1}{r}\left(\psi^{\prime}+g \xi_{r}+\frac{\delta P}{\rho}\right)$.
The equation of mass conservation together with Eq. (3) gives:

$\frac{\delta \rho}{\rho}+\frac{1}{r^{2}} \frac{\partial}{\partial r}\left(r^{2} \xi_{r}\right)-\frac{\ell(\ell+1)}{\omega^{2} r^{2}}\left(\psi^{\prime}+g \xi_{r}+\frac{\delta P}{\rho}\right)=0$.

The perturbed Poisson equation reads:

$\frac{1}{r^{2}} \frac{\partial}{\partial r}\left(r^{2} \frac{\partial \psi^{\prime}}{\partial r}\right)-\frac{\ell(\ell+1)}{r^{2}} \psi^{\prime}=4 \pi G\left(\delta \rho-\frac{\mathrm{d} \rho}{\mathrm{d} r} \xi_{r}\right)$.

And finally, the equation of energy conservation reads:

$$
\begin{aligned}
\mathrm{i} \omega T c_{\mathrm{v}}\left(\frac{\delta S}{c_{\mathrm{v}}}\right)= & -\frac{\partial \delta L_{r}}{\partial M_{r}}+\epsilon\left(\frac{\delta \epsilon}{\epsilon}+\frac{\delta \rho}{\rho}+\frac{1}{r^{2}} \frac{\partial\left(r^{2} \xi_{r}\right)}{\partial r}\right) \\
& +\ell(\ell+1) \frac{L}{4 \pi \rho r^{3}}\left(\frac{\delta T}{r \mathrm{~d} T / \mathrm{d} r}-\frac{\xi_{r}}{r}\right),
\end{aligned}
$$

where $L_{r}=4 \pi r^{2} F_{r}$. In the diffusion approximation, $\delta L_{r} / L$ is given by:

$\frac{\delta L_{r}}{L}=2 \frac{\xi_{r} v v}{r}+3 \frac{\delta T}{T}-\frac{\delta \kappa}{\kappa}-\frac{\delta \rho}{\rho}+\frac{\partial \delta T / \partial r}{\mathrm{~d} T / \mathrm{d} r}-\frac{\partial \xi_{r}}{\partial r}$.

\section{Treatment of the atmosphere}

The system of equations used in the interior is no longer valid in the atmosphere, for two reasons. Firstly, the diffusion equation relating the radiative flux to the local gradient of temperature is no longer valid, and secondly, the interior approximation of the radiative pressure

$P_{\mathrm{R}}=\frac{1}{3} a T^{4}$

is also no longer valid in the atmosphere. In Sect. 3.1, we explain how Eqs. (6) and (7) can be replaced by a more appropriate one. Avoiding Eq. (8) will require a rewriting of the momentum equation, as explained in Sect. 3.2. In Sect. 3.3, we give the surface boundary conditions used to close the problem.

\subsection{Radiative equilibrium in the local atmosphere}

In a non-radially pulsating star, all the eigenfunctions have an angular dependence. It is therefore useful to define a "local atmosphere" as the gas column at a given angular position $(\theta, \varphi)$, and at a given time. In what follows we will always refer to this local atmosphere although we will often omit the word "local" for the sake of brevity.

An important property of the atmosphere of a pulsating star is that its heat capacity is very small. Defining the thermal relaxation time of the atmosphere as the time necessary for it to lose all its internal energy with a luminosity $L$ :

$\tau_{\mathrm{th}}=\int_{\mathrm{atm} .} T c_{\mathrm{v}} \mathrm{d} m / L$

we find typical values $\tau_{\text {th }} \approx 1 \mathrm{~s}$ for the atmosphere of $\beta$ Cephei as well as Slowly Pulsating B stars (SPBs), which is much smaller than their typical pulsation periods. In a 
very good approximation, we can therefore assume that, for $\beta$ Cephei stars and SPBs, the local atmosphere remains in radiative equilibrium during the pulsation $(\nabla \cdot \boldsymbol{F}=0)$.

A hydrostatic equilibrium atmosphere model in the plane-parallel approximation is entirely determined by its effective temperature $T_{\text {eff }}$, its gravity $g$ and its chemical composition. Given the chemical composition, the local temperature can therefore be written as a function of the Rosseland mean optical depth $\tau, T_{\text {eff }}$ and $g$ :

$T=T\left(\tau, T_{\mathrm{eff}}, g\right)$.

Considering the radiative equilibrium property of the local atmosphere, our main approximation is to assume that, at each phase during the pulsation cycle, the $T(\tau)$ law in the local atmosphere is the same as the $T(\tau)$ law of an equilibrium atmosphere model. During the pulsation, the two parameters $T_{\text {eff }}$ and $g$ characterising the equilibrium atmosphere model, and thus the local atmosphere, are varying with time and are function of $\theta$ and $\varphi$. For a given time and a given $(\theta, \varphi)$, the temperature in the local atmosphere (equilibrium value + perturbation) is thus given by:

$T_{0}+\delta T=T\left(\tau_{0}+\delta \tau, T_{\text {eff } 0}+\delta T_{\text {eff }}, g_{0}+\delta g_{\mathrm{e}}\right)$,

where $\delta \tau$ is the Lagrangian perturbation of the Rosseland mean optical depth; we remark that layers of constant optical depth do not follow the motion of matter. $\delta T_{\text {eff }}$ is the variation of the effective temperature of the local atmosphere. $\delta g_{\mathrm{e}}$ is the variation of the local gravity from the point of view of a comoving frame, i.e. the gravity corrected for the pulsational acceleration.

In the linear approximation, Eq. (11) gives:

$$
\frac{\delta T}{T}=\frac{\partial \ln T}{\partial \ln T_{\mathrm{eff}}} \frac{\delta T_{\mathrm{eff}}}{T_{\mathrm{eff}}}+\frac{\partial \ln T}{\partial \ln g_{\mathrm{e}}} \frac{\delta g_{\mathrm{e}}}{g_{\mathrm{e}}}+\frac{\partial \ln T}{\partial \ln \tau} \frac{\delta \tau}{\tau} .
$$

For a given mode, the different perturbed quantities appearing in this equation are proportional to the spherical harmonic $Y_{\ell}^{m}(\theta, \varphi)$. Perturbing the definition of the Rosseland mean optical depth leads to:

$\frac{\partial \delta \tau}{\partial \tau}=\frac{\delta \kappa}{\kappa}+\frac{\delta \rho}{\rho}+\frac{\partial \xi_{r}}{\partial r}$.

And finally, eliminating $\delta \tau$ between Eq. (12) and Eq. (13) gives:

$$
\begin{aligned}
\frac{\partial(\delta T / T)}{\partial \ln \tau}= & \frac{\partial \ln T}{\partial \ln \tau}\left(\frac{\delta \kappa}{\kappa}+\frac{\delta \rho}{\rho}+\frac{\partial \xi_{r}}{\partial r}\right) \\
- & \left(1-\frac{\partial^{2} \ln T / \partial \ln \tau^{2}}{\partial \ln T / \partial \ln \tau}\right) \\
& \left(\frac{\delta T}{T}-\frac{\partial \ln T}{\partial \ln T_{\mathrm{eff}}} \frac{\delta T_{\mathrm{eff}}}{T_{\mathrm{eff}}}-\frac{\partial \ln T}{\partial \ln g_{\mathrm{e}}} \frac{\delta g_{\mathrm{e}}}{g_{\mathrm{e}}}\right) \\
+ & \frac{\partial^{2} \ln T}{\partial \ln \tau \partial \ln T_{\mathrm{eff}}} \frac{\delta T_{\mathrm{eff}}}{T_{\mathrm{eff}}}+\frac{\partial^{2} \ln T}{\partial \ln \tau \partial \ln g_{\mathrm{e}}} \frac{\delta g_{\mathrm{e}}}{g_{\mathrm{e}}}
\end{aligned}
$$

It is Eq. (14) that we use instead of Eqs. (6) and (7) in the atmosphere. The different derivatives appearing in this equation are numerically estimated using a family of hydrostatic equilibrium atmosphere models with different effective temperatures and gravities bracketing those of the reference equilibrium model around which the star is oscillating. In our applications, we use the atmosphere models by Kurucz (1993). How we compute $\delta T_{\text {eff }} / T_{\text {eff }}$ and $\delta g_{\mathrm{e}} / g_{\mathrm{e}}$ is explained in Sect. 3.3.

\subsection{Acceleration due to the radiation}

In the previous section, we argued that the temperature distribution of the local atmosphere can be obtained from an equilibrium atmosphere model. The pressure and density distributions as well as the displacement vector, however, must be computed by solving the equations of conservation of mass and momentum in the entire atmosphere. In what follows we explain how this is done.

In the outer atmosphere of a star, the radiation field is no longer isotropic. As a consequence, the radiation pressure tensor cannot be represented anymore by a diagonal matrix with constant elements and the law $P_{\mathrm{R}}=$ $(1 / 3) a T^{4}$ is no longer valid (see e.g. Mihalas \& WeibelMihalas 1999, Sect. 66). We recall that the equilibrium quantities we use in the atmosphere are obtained from the models of Kurucz (1993). In these models, the total pressure gradient is split up into the gas pressure gradient and the acceleration due to the radiation (see Kurucz 1970, Sect. 2.11). In the perturbed model, we proceed in the same way, which permits to keep the consistency with the equilibrium model. The momentum equation reads then:

$\frac{\partial \boldsymbol{v}}{\partial t}+\boldsymbol{v} \cdot \nabla \boldsymbol{v}=-\nabla \psi-\frac{\nabla P_{\mathrm{g}}}{\rho}+\boldsymbol{a}_{\mathbf{R}}$

where $\boldsymbol{v}$ is the velocity, $P_{\mathrm{g}}$ is the gas pressure and $\boldsymbol{a}_{\mathbf{R}}$ is the acceleration vector due to the radiation. In this case, the flux-weighted mean opacity $\kappa_{\mathrm{F}}$ is very useful since it can relate directly the acceleration due to the radiation to the flux:

$\boldsymbol{a}_{\mathbf{R}}=\kappa_{\mathrm{F}} \boldsymbol{F} / c$,

(see e.g. Mihalas \& Weibel-Mihalas 1999, Sect. 82). To obtain the perturbed momentum equation, we make the following assumptions:

a1) We assume that $\delta|\boldsymbol{F}|$ remains constant from the base of the atmosphere to the outermost layer.

a2) We assume that the flux vector $\boldsymbol{F}$ is parallel with $-\nabla T$ during the entire pulsation cycle.

a3) We approximate the relative variation of the fluxweighted mean opacity by the relative variation of the Rosseland opacity: $\delta \kappa_{\mathrm{F}} / \kappa_{\mathrm{F}} \simeq \delta \kappa / \kappa$.

To justify assumption (a1), we recall that the equilibrium atmosphere models we use (Kurucz models) are obtained assuming that the atmosphere is in radiative equilibrium and that it is so thin that a plane parallel approximation can be adopted. These two hypotheses imply that the equilibrium flux is assumed to be constant. As pointed out in 
Sect. 3.1, the very small thermal relaxation time of the atmosphere permits us to assume that the local atmosphere remains in radiative equilibrium during the pulsation. This leads us to assume, for the same reasons as in equilibrium, that $\delta|\boldsymbol{F}|$ remains constant in the atmosphere. Under the diffusion approximation, assumptions (a2) and (a3) are clearly valid (see e.g. Mihalas \& WeibelMihalas 1999, Sect. 82 for the equivalence between the two mean opacities). Assumptions (a2) and (a3) do not imply that the diffusion approximation is valid, because we adopt the equilibrium values of $\boldsymbol{a}_{\mathbf{R}}$ given by the atmosphere models of Kurucz, instead of obtaining them by computing the derivative of Eq. (8). It is not easy to examine to what extent assumptions (a2) and (a3) remain valid in the outermost layers of the atmosphere. We did different tests by changing artificially the coefficients of the equations associated to these assumptions and the conclusion is that the errors coming from them have a negligible impact on the final results of our study.

Assumption (a1) together with Stefan's law gives at first order:

$\frac{\delta|\boldsymbol{F}|}{|\boldsymbol{F}|}=\frac{\delta F_{r}}{F_{r}}=4 \frac{\delta T_{\mathrm{eff}}}{T_{\mathrm{eff}}}$.

Then, assumption (a3) together with Eq. (16) gives, for the radial component of $\delta \boldsymbol{a}_{\mathbf{R}}$ :

$\left(\delta \boldsymbol{a}_{\mathbf{R}}\right)_{r}=a_{\mathrm{R}}\left(\frac{\delta \kappa}{\kappa}+4 \frac{\delta T_{\mathrm{eff}}}{T_{\mathrm{eff}}}\right)$.

In the following equations, $a_{\mathrm{R}}$ corresponds to the equilibrium value of the acceleration due to the radiation. This quantity is obtained at each layer directly from the Kurucz atmosphere models. From Eqs. (15) and (18), the radial component of the perturbed momentum equation reads finally:

$$
\begin{aligned}
\omega^{2} \xi_{r}= & \frac{\partial\left(\delta P_{\mathrm{g}} / P_{\mathrm{g}}\right)}{\partial r} \frac{P_{\mathrm{g}}}{\rho} \\
& +\frac{\partial \psi^{\prime}}{\partial r}+\frac{\partial\left(g \xi_{r}\right)}{\partial r}+\left(\frac{\delta \rho}{\rho}-\frac{\delta P_{\mathrm{g}}}{P_{\mathrm{g}}}\right)\left(g-a_{\mathrm{R}}\right) \\
& -a_{\mathrm{R}}\left(\frac{\delta \kappa}{\kappa}+4 \frac{\delta T_{\mathrm{eff}}}{T_{\mathrm{eff}}}+\frac{\partial \xi_{r}}{\partial r}\right) .
\end{aligned}
$$

Assumption (a2) is used to compute the transversal component of the momentum equation, and we find:

$\omega^{2} \xi_{h}=\frac{1}{r}\left(\frac{\delta P_{\mathrm{g}}}{\rho}+\psi^{\prime}+g \xi_{r}-a_{\mathrm{R}} \frac{\delta T}{\partial T / \partial r}\right)$.

Consequently, the continuity equation reads:

$$
\begin{aligned}
\frac{\delta \rho}{\rho}+ & \frac{1}{r^{2}} \frac{\partial}{\partial r}\left(r^{2} \xi_{r}\right)= \\
& \frac{\ell(\ell+1)}{\omega^{2} r^{2}}\left(\frac{\delta P_{\mathrm{g}}}{\rho}+\psi^{\prime}+g \xi_{r}-\frac{a_{\mathrm{R}} \delta T}{\partial T / \partial r}\right) .
\end{aligned}
$$

Equations. (19) and (21) are used in the atmosphere instead of Eqs. (2) and (4). In the following subsection, we will show that these two sets of equations coincide at the connecting layer between interior and atmosphere.

\subsection{Boundary and matching conditions}

The matching conditions we impose are the continuity of the different perturbed variables at the connecting layer between the interior and the atmosphere. The matching condition imposed on the perturbed flux allows us to compute $\delta T_{\text {eff }} / T_{\text {eff }}$. Indeed, from Eqs. (7) and (17), we find at the connecting layer:

$3 \frac{\delta T}{T}-\frac{\delta \kappa}{\kappa}-\frac{\delta \rho}{\rho}+\frac{\partial \delta T / \partial r}{\mathrm{~d} T / \mathrm{d} r}-\frac{\partial \xi_{r}}{\partial r}=4 \frac{\delta T_{\mathrm{eff}}}{T_{\mathrm{eff}}}$.

Also $\delta g_{\mathrm{e}} / g_{\mathrm{e}}$ is obtained at the connecting layer. The variation of the gravity from the point of view of a comoving frame reads:

$\delta g_{\mathrm{e}}=-\left(\delta \boldsymbol{g}_{\mathbf{e}}\right)_{r}=\delta(\partial \psi / \partial r)-\omega^{2} \xi_{r}$

After some simple developments, we find then:

$\frac{\delta g_{\mathrm{e}}}{g_{\mathrm{e}}}=\frac{\partial \psi^{\prime} / \partial r}{g}+\frac{4 \pi \rho r^{3}}{M_{r}} \frac{\xi_{r}}{r}-\left(2+\frac{\omega^{2} r}{g}\right) \frac{\xi_{r}}{r}$.

And we note that under the Cowling approximation and neglecting the surface density divided by the mean density of the star, this equation takes the very simple form:

$\frac{\delta g_{\mathrm{e}}}{g_{\mathrm{e}}}=-\left(2+\frac{\omega^{2} r}{g}\right) \frac{\xi_{r}}{r}$.

It is important to realize that Eqs. (19) and (21) mathematically coincide with Eqs. (2) and (4) at the connecting layer. This follows directly from Eqs. (8) and (22). As a consequence, the derivatives of $\xi_{r} / R$ and $\delta P_{\mathrm{g}} / P_{\mathrm{g}}$ are continuous at the connecting layer. The continuity of the derivative of $\delta T / T$ at the connecting layer is not imposed by the equations. For all the modes we have computed, the smoothness of $\delta T / T$ at the connecting layer was found a posteriori (see Figs. 4 and 5). This confirms the validity of our treatment.

Different boundary conditions have to be imposed at the outermost layer of the model. Firstly, we have the mechanical boundary condition. Usually, the mechanical boundary condition is obtained by assuming that the third term of the right hand side of Eq. (2) goes to zero at the surface. This condition is justified either by assuming that $\lim _{\tau \rightarrow 0} P /(\rho g R)=0$ (Cox 1980, Sect. 17.6b) or by assuming that $\partial(\delta P / P) / \partial r=0$ at the surface (Pesnell 1990). However, for massive stars such as $\beta$ Cephei stars and SPBs, the acceleration due to the radiation $\left(\boldsymbol{a}_{\mathbf{R}}\right)$ cannot be neglected in Eq. (15). Our mechanical boundary condition is obtained by neglecting the contribution due to the gas pressure alone. More precisely, we neglect the first term of Eq. (19) at the surface, and the mechanical boundary condition is:

$$
\begin{aligned}
\omega^{2} \xi_{r}= & \frac{\partial \psi^{\prime}}{\partial r}+\frac{\partial\left(g \xi_{r}\right)}{\partial r}+\left(\frac{\delta \rho}{\rho}-\frac{\delta P_{\mathrm{g}}}{P_{\mathrm{g}}}\right)\left(g-a_{\mathrm{R}}\right) \\
& -a_{\mathrm{R}}\left(\frac{\delta \kappa}{\kappa}+4 \frac{\delta T_{\mathrm{eff}}}{T_{\mathrm{eff}}}+\frac{\partial \xi_{r}}{\partial r}\right) .
\end{aligned}
$$

The potential boundary condition is, as usual, obtained by imposing a first order continuous match (i.e. continuity of 
$\psi^{\prime}$ and its first derivatives) between the inner solution of the Poisson equation and the outer solution of the Laplace equation (Ledoux \& Walraven 1958):

$\frac{\partial \psi^{\prime}}{\partial r}+\frac{\ell+1}{r} \psi^{\prime}=-4 \pi G \rho \xi_{r}$

Finally, also Eq. (14) needs a boundary condition. It is obtained by evaluating Eq. (12) at the outermost layer. Using the rule of l'Hospital and Eq. (13) to evaluate $\lim _{\tau \rightarrow 0} \delta \tau / \tau$, we obtain the following boundary condition at the surface:

$$
\begin{aligned}
\frac{\delta T}{T}= & \frac{\partial \ln T}{\partial \ln T_{\mathrm{eff}}} \frac{\delta T_{\mathrm{eff}}}{T_{\mathrm{eff}}}+\frac{\partial \ln T}{\partial \ln g_{\mathrm{e}}} \frac{\delta g_{\mathrm{e}}}{g_{\mathrm{e}}} \\
& +\frac{\partial \ln T}{\partial \ln \tau}\left(\frac{\delta \kappa}{\kappa}+\frac{\delta \rho}{\rho}+\frac{\partial \xi_{r}}{\partial r}\right) .
\end{aligned}
$$

\subsection{Comparison with other approximations}

To place our approximation in a broader context, we first recall the basic approximations made in three different equilibrium atmosphere models: the Eddington atmosphere, the grey atmosphere and the non-grey (e.g. Kurucz) atmosphere.

The Eddington atmosphere is a plane-parallel, grey atmosphere, in radiative equilibrium and in LTE, where it is assumed that $J=3 K(J$ is the mean intensity and $K$ is the second angular moment of the radiation field). Its temperature distribution obeys the following well known law:

$T^{4}(\tau)=\frac{3}{4} T_{\mathrm{eff}}^{4}\left(\tau+\frac{2}{3}\right)$

For a more general grey atmosphere, it is not assumed that $J=3 \mathrm{~K}$, and the temperature distribution is given by

$T^{4}(\tau)=\frac{3}{4} T_{\mathrm{eff}}^{4}(\tau+q(\tau))$

where $q(\tau)$ is the well known Hopf function, which can be determined analytically as well as numerically (see e.g. Mihalas 1978). We note that the Hopf function is unique, it does not depend on the effective temperature and gravity of the atmosphere. Finally, if we consider much more precise non-grey atmospheres (such as the models of $\mathrm{Ku}$ rucz), the temperature distribution takes a general form given by Eq. (10). We emphasize that non-grey Kurucz atmospheres differ significantly from Eddington and grey atmospheres. In Fig. 1, we compare the temperature distribution of a Kurucz atmosphere to the one of an Eddington atmosphere for a $10 M_{\odot} \beta$ Cephei model. We see that the two distributions are very different in most of the atmosphere. In particular, the steep temperature gradient found up to very small optical depths in the Kurucz atmosphere is not present in the Eddington atmosphere.

Our method is easily understood by following the same line of reasoning, going from Eddington atmospheres to non-grey atmospheres, in the case of pulsating stars. More

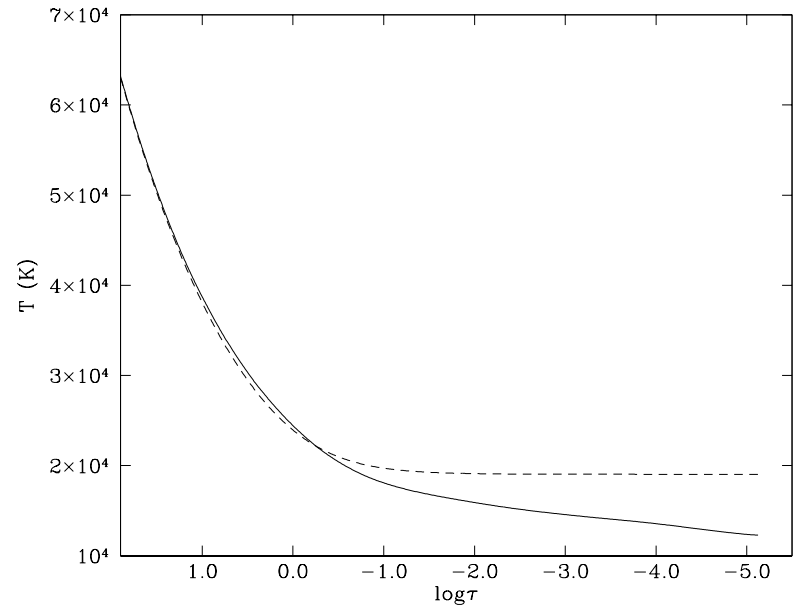

Fig. 1. Temperature distribution of a Kurucz atmosphere model (solid line) compared to the one of an Eddington model (dashed line), in the atmosphere of a $10 M_{\odot} \beta$ Cephei model. The abscissa corresponds to the logarithm of the Rosseland optical depth.

precisely, in a pulsating star, the Eddington approximation leads to the following equations (Saio \& Cox 1980; Balmforth 1992):

$$
\begin{aligned}
\boldsymbol{F} & =-\frac{4 \pi}{3(\kappa+\sigma) \rho} \nabla J, \\
J & =B+\frac{T}{4 \pi \kappa} \frac{\mathrm{d} S}{\mathrm{~d} t} .
\end{aligned}
$$

In a plane-parallel atmosphere at radiative equilibrium, this system of equations reduces to Eq. (29) (see e.g. Mihalas \& Weibel-Mihalas 1999, Sect. 82). In particular, we note that the hypothesis of hydrostatic equilibrium need not be made in order to obtain Eqs. (29) and (30). In the beginning of Sect. 3.1, we argued that the hypothesis of radiative equilibrium can be applied to the perturbed atmosphere, and the plane-parallel hypothesis is certainly acceptable in our applications. Therefore, adopting the Eddington approximation in the atmosphere of a pulsating star reduces in very good approximation to perturbing Eq. (29). For the same physical reasons, making the grey approximation in the atmosphere of a pulsating star leads simply to perturbing Eq. (30), an approach which is adopted by Dupret (2001). In this paper, we proceed in the same way, but now for the more realistic non-grey atmosphere models, which leads us to perturb Eq. (10).

We conclude that the Eddington approximation as well as the grey atmosphere approximation are particular cases of the more general approach presented in Sect. 3.1. As the Eddington and grey atmospheres do not lead to a good temperature distribution in the outer layers of the atmosphere (see Fig. 1), the gain is significant when using more realistic non-grey atmosphere models as we do. It is important to note that, in the general method of small perturbations, the use of an approximation (e.g. the Eddington approximation) in the perturbed model makes sense if and only if it is made in the equilibrium model as well. Therefore, one of the main advantages of our method 
is simply that it permits to use better equilibrium models (the Kurucz atmosphere models).

It could be argued that consistency is somehow lost in our method, because the Kurucz atmosphere models make explicitly the hypothesis of hydrostatic equilibrium, which is not valid for pulsating stars. We argue however, that the gain with our method is significant. First, the apparent loss of consistency is not important for SPB high order g-modes and $\beta$ Cephei low order p-modes. If we compare, for example, the values of $\delta P_{\mathrm{g}} / P_{\mathrm{g}}$ obtained by our method (solving the momentum equations throughout the entire atmosphere) to the ones consistent with the static Kurucz models, we find relative differences between 10 and $20 \%$, depending on the mode order. Secondly, the coupling between the thermal structure of the atmosphere $\left(T(\tau)\right.$ and $\left.T_{\text {eff }}\right)$ and its dynamical structure (linked in first approximation to its effective gravity) is generally small. The coefficient $\partial \ln T / \partial \ln g_{\mathrm{e}}$ appears to be much smaller than $\partial \ln T / \partial \ln T_{\text {eff }}$, and even with the significant values of $\delta g_{\mathrm{e}} / g_{\mathrm{e}}$ found for the $\beta$ Cephei p-modes, the corresponding term in Eq. (12) remains much smaller than the two other ones (see Fig. 4 in Sect. 4). It is only for high-order p-modes with frequencies close to the acoustic cut-off, that our use of hydrostatic Kurucz models becomes more questionable. For such modes, the only rigorous way would be to solve explicitly the perturbed equations of radiation hydrodynamics, which is beyond the scope of our current study.

We do not claim that the use of the Eddington and diffusion approximations are inappropriate in the study of non-adiabatic oscillations. For the study of the excitation and damping mechanisms in pulsating stars, these approximations remain perfectly appropriate since these mechanisms occur in layers much deeper than the atmosphere. Similarly, the adiabatic approximation remains suitable for the computation of the frequencies of g-modes and moderate order p-modes, as they are determined mostly by the internal layers.

As a final remark, we note that a better thermal boundary condition has been proposed by Gabriel (1989). The problem with Gabriel's treatment is that it applies only to the very superficial layers of a star, where matter and radiation no longer interact, but no treatment is proposed between the photosphere and these very superficial layers.

\section{Results}

As an illustration, we present in this section some of the results obtained for a typical $\beta$ Cephei model and for a typical SPB model.

The $\beta$ Cephei equilibrium model is near the TAMS and it has the following characteristics:

$$
\begin{array}{lll}
M=10 M_{\odot} & \log (g)=3.76 \\
T_{\text {eff }}=22643 \mathrm{~K} & X & =0.7 \\
L=11339 L_{\odot} & Z & =0.02 \\
R=6.93 R_{\odot} & \text { Age } & =18.58 \mathrm{Myr} .
\end{array}
$$

Table 1. Properties of non-adiabatic eigenfunctions in the atmosphere for different $p$-modes of a $10 M_{\odot}$ model ( $\beta$ Cephei). $\left|\delta T_{\text {eff }} / T_{\text {eff }}\right|$ is the modulus of $\delta T_{\text {eff }} / T_{\text {eff }}, \phi_{T}$ is the phase difference between $\delta T_{\text {eff }} / T_{\text {eff }}$ and $\xi_{r}$ in degrees, $\left(\delta g_{\mathrm{e}} / g_{\mathrm{e}}\right)_{\Re}$ is the real part of $\delta g_{\mathrm{e}} / g_{\mathrm{e}},\left(\xi_{h} / \xi_{r}\right)_{\Re}$ is the real part of $\xi_{h} / \xi_{r}$ and $P$ is the period in hours. The eigenfunctions are normalized so that $\xi_{r} / R=1$ at $\tau=2 / 3$.

\begin{tabular}{llllllll}
\hline \hline \multicolumn{1}{c}{ mode } & $\left|\frac{\delta T_{\text {eff }}}{T_{\text {eff }}}\right|$ & $\phi_{T}\left(^{\circ}\right)$ & $\left(\frac{\delta g_{\mathrm{e}}}{g_{\mathrm{e}}}\right)_{\Re}$ & $\left(\frac{\xi_{h}}{\xi_{r}}\right)_{\Re}$ & $\frac{G M}{\omega^{2} R^{3}}$ & $P(\mathrm{~h})$ \\
\hline$\ell=1$ & $\mathrm{p}_{1}$ & 3.07 & 179 & -22.02 & 0.048 & 0.049 & 3.56 \\
$\ell=1$ & $\mathrm{p}_{2}$ & 3.48 & -173 & -28.52 & 0.036 & 0.037 & 3.09 \\
$\ell=1$ & $\mathrm{p}_{3}$ & 3.86 & -165 & -36.42 & 0.027 & 0.028 & 2.71 \\
$\ell=2$ & $\mathrm{f}$ & 3.48 & -174 & -28.16 & 0.036 & 0.038 & 3.11 \\
$\ell=2$ & $\mathrm{p}_{1}$ & 3.71 & -169 & -32.57 & 0.031 & 0.032 & 2.87 \\
$\ell=2$ & $\mathrm{p}_{2}$ & 4.02 & -161 & -40.63 & 0.024 & 0.025 & 2.55 \\
$\ell=2$ & $\mathrm{p}_{3}$ & 4.23 & -148 & -55.04 & 0.017 & 0.018 & 2.17 \\
$\ell=3$ & $\mathrm{f}$ & 3.69 & -170 & -31.71 & 0.032 & 0.033 & 2.92 \\
$\ell=3$ & $\mathrm{p}_{1}$ & 4.13 & -158 & -44.54 & 0.021 & 0.023 & 2.43 \\
$\ell=3$ & $\mathrm{p}_{2}$ & 4.25 & -151 & -51.60 & 0.018 & 0.020 & 2.24 \\
$\ell=3$ & $\mathrm{p}_{3}$ & 4.23 & -144 & -60.31 & 0.015 & 0.017 & 2.06 \\
$\ell=4$ & $\mathrm{f}$ & 3.92 & -165 & -36.40 & 0.027 & 0.029 & 2.71 \\
$\ell=4$ & $\mathrm{p}_{1}$ & 4.21 & -155 & -47.98 & 0.020 & 0.021 & 2.33 \\
$\ell=4$ & $\mathrm{p}_{2}$ & 4.19 & -141 & -64.45 & 0.014 & 0.015 & 1.99 \\
$\ell=5$ & $\mathrm{f}$ & 4.25 & -155 & -48.04 & 0.020 & 0.021 & 2.33 \\
$\ell=5$ & $\mathrm{p}_{1}$ & 4.27 & -152 & -51.25 & 0.018 & 0.020 & 2.25 \\
$\ell=5$ & $\mathrm{p}_{2}$ & 4.13 & -138 & -68.22 & 0.013 & 0.015 & 1.93 \\
\hline
\end{tabular}

The SPB equilibrium model is halfway between the ZAMS and the TAMS and it has the following characteristics:

$$
\begin{array}{lll}
M=5 M_{\odot} & \log (g)=3.93 \\
T_{\text {eff }}=15190 \mathrm{~K} & X & =0.7 \\
L=765 L_{\odot} & Z & =0.02 \\
R=4.00 R_{\odot} & \text { Age } & =59.07 \text { Myr. }
\end{array}
$$

In these two models, $R$ is by definition the radius at $\tau=2 / 3$. We recall that in our models, the outermost layer of the atmosphere corresponds to a much smaller optical depth $(\log \tau=-4.125)$.

In Tables 1 and 2, we give some of the results obtained for different modes in the atmosphere. Table 1 corresponds to the $\beta$ Cephei model and Table 2 to the SPB model. All the eigenfunctions are normalized so that $\xi_{r} / R=1$ at $\tau=2 / 3$.

In the second column, we give $\left|\delta T_{\text {eff }} / T_{\text {eff }}\right|$. For the $\beta$ Cephei model, the values obtained are relatively small. For the SPB model, we see that $\left|\delta T_{\text {eff }} / T_{\text {eff }}\right|$ increases quickly as the order of the mode increases.

In the third column, we give the phase difference between $\delta T_{\text {eff }} / T_{\text {eff }}$ and $\xi_{r}$ in degrees $\left(\phi_{T}\right)$. We recall that in the adiabatic case, the temperature variations are in opposite phase with the radial displacement for $\beta$ Cephei p-modes and on the contrary, that they are in phase with the radial displacement for SPB g-modes. Due to the nonadiabaticity of the superficial layers, the values of $\phi_{T}$ we obtain are no longer exactly $\pm 180^{\circ}$ or $0^{\circ}$. Comparing $\phi_{T}$ to 
Table 2. Properties of non-adiabatic eigenfunctions in the atmosphere for different $g$-modes of a $5 M_{\odot}$ model (SPB), same quantities as in Table 1.

\begin{tabular}{llllllll}
\hline \hline \multicolumn{1}{c}{ mode } & $\left|\frac{\delta T_{\text {eff }}}{T_{\text {eff }}}\right|$ & $\phi_{T}\left(^{\circ}\right)$ & $\left(\frac{\delta g_{\mathrm{e}}}{g_{\mathrm{e}}}\right)_{\Re}$ & $\left(\frac{\xi_{h}}{\xi_{r}}\right)_{\Re}$ & $\frac{G M}{\omega^{2} R^{3}}$ & $P(\mathrm{~h})$ \\
\hline$\ell=1$ & $\mathrm{~g}_{20}$ & 3.66 & -41 & -2.02 & 17.77 & 17.29 & 41.36 \\
$\ell=1$ & $\mathrm{~g}_{25}$ & 5.78 & -33 & -1.99 & 25.02 & 24.09 & 48.82 \\
$\ell=1$ & $\mathrm{~g}_{30}$ & 8.80 & -24 & -1.95 & 36.87 & 34.86 & 58.73 \\
$\ell=1$ & $\mathrm{~g}_{35}$ & 11.72 & -17 & -1.94 & 51.44 & 47.16 & 68.31 \\
$\ell=1$ & $\mathrm{~g}_{40}$ & 14.28 & -11 & -1.89 & 69.15 & 62.10 & 78.38 \\
$\ell=1$ & $\mathrm{~g}_{45}$ & 16.48 & -6.2 & -1.85 & 90.11 & 78.47 & 88.11 \\
$\ell=1$ & $\mathrm{~g}_{50}$ & 18.29 & -2.6 & -1.79 & 114.6 & 96.78 & 97.85 \\
$\ell=2$ & $\mathrm{~g}_{20}$ & 2.06 & -46 & -2.14 & 5.95 & 5.80 & 23.96 \\
$\ell=2$ & $\mathrm{~g}_{25}$ & 4.23 & -39 & -2.07 & 9.01 & 8.65 & 29.25 \\
$\ell=2$ & $\mathrm{~g}_{30}$ & 7.15 & -31 & -2.01 & 13.18 & 12.40 & 35.03 \\
$\ell=2$ & $\mathrm{~g}_{35}$ & 10.30 & -23 & -1.96 & 18.39 & 16.88 & 40.86 \\
$\ell=2$ & $\mathrm{~g}_{40}$ & 13.07 & -17 & -1.91 & 24.12 & 21.56 & 46.18 \\
$\ell=2$ & $\mathrm{~g}_{45}$ & 15.88 & -11 & -1.87 & 31.79 & 27.45 & 52.11 \\
$\ell=2$ & $\mathrm{~g}_{50}$ & 18.29 & -6.6 & -1.82 & 40.73 & 33.84 & 57.86 \\
$\ell=2$ & $\mathrm{~g}_{55}$ & 20.32 & -3.1 & -1.78 & 50.68 & 40.41 & 63.23 \\
$\ell=2$ & $\mathrm{~g}_{60}$ & 22.38 & 0.1 & -1.72 & 64.05 & 48.46 & 69.24 \\
$\ell=3$ & $\mathrm{~g}_{20}$ & 1.14 & -30 & -2.30 & 3.00 & 2.93 & 17.02 \\
$\ell=3$ & $\mathrm{~g}_{25}$ & 2.78 & -35 & -2.18 & 4.54 & 4.35 & 20.75 \\
$\ell=3$ & $\mathrm{~g}_{30}$ & 5.27 & -32 & -2.09 & 6.61 & 6.22 & 24.81 \\
$\ell=3$ & $\mathrm{~g}_{35}$ & 8.32 & -27 & -2.02 & 9.23 & 8.47 & 28.95 \\
$\ell=3$ & $\mathrm{~g}_{40}$ & 11.23 & -21 & -1.96 & 12.10 & 10.81 & 32.71 \\
$\ell=3$ & $\mathrm{~g}_{45}$ & 14.37 & -15 & -1.90 & 15.92 & 13.75 & 36.88 \\
$\ell=3$ & $\mathrm{~g}_{50}$ & 17.18 & -10 & -1.85 & 20.41 & 16.95 & 40.96 \\
$\ell=3$ & $\mathrm{~g}_{55}$ & 19.58 & -5.8 & -1.80 & 25.39 & 20.24 & 44.75 \\
$\ell=3$ & $\mathrm{~g}_{60}$ & 22.02 & -2.0 & -1.73 & 32.06 & 24.25 & 48.98 \\
$\ell=3$ & $\mathrm{~g}_{65}$ & 24.33 & 1.0 & -1.67 & 40.06 & 28.55 & 53.15 \\
\hline
\end{tabular}

the adiabatic phase difference shows that the extra phase lag increases as the order of the mode increases for the $\beta$ Cephei p-modes and on the contrary, that the extra phase lag decreases as the order of the mode increases for the SPB g-modes.

In the fourth column, we give the real part of $\delta g_{\mathrm{e}} / g_{\mathrm{e}}$. The imaginary part turns out to be always negligible. We recall that $\delta g_{\mathrm{e}} / g_{\mathrm{e}}$ is the Lagrangian variation of the gravity from the point of view of a comoving frame. We see that, for the $\beta$ Cephei model, the values obtained increase quickly with the order of the mode. This comes from the increasing frequency and therefore increasing acceleration of the matter for a fixed displacement amplitude - term $\omega^{2} \xi_{r}$ in Eq. (23). Although our method is appropriate for $\mathrm{g}$-modes and moderate-order p-modes, this rapid increase of the amplitude of $\delta g_{\mathrm{e}} / g_{\mathrm{e}}$ with frequency leads us to suspect that our approach becomes more questionable for high-order p-modes with frequencies close to the acoustic cut-off. For the SPB g-modes, we see that $\delta g_{\mathrm{e}} / g_{\mathrm{e}}$ remains very small.

In the fifth column, we give the real part of $\xi_{h} / \xi_{r}$ at $\tau=2 / 3$, and in the sixth column, we give the real part of $G M /\left(R^{3} \omega^{2}\right)$, which is sometimes used to approximate $\xi_{h} / \xi_{r}$. The imaginary part of $\xi_{h} / R$, and thus the

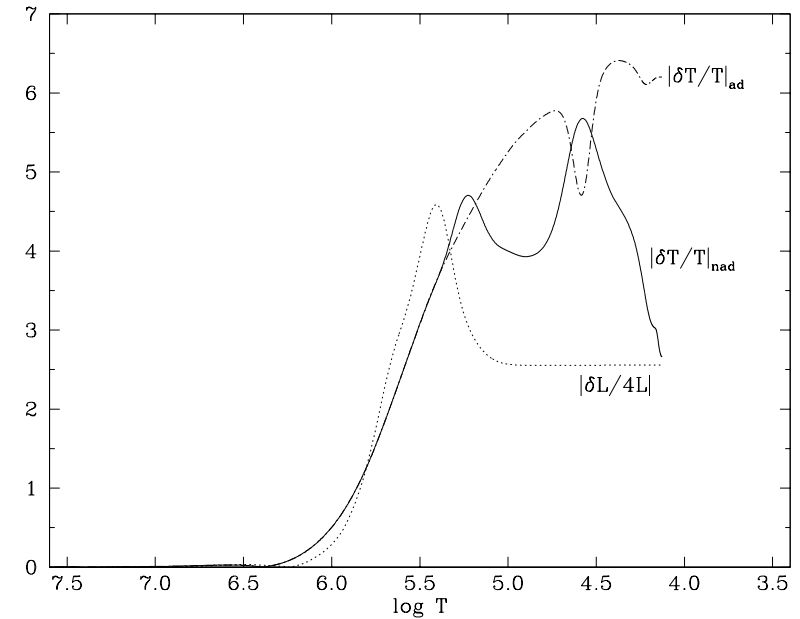

Fig. 2. Amplitude of the non-adiabatic eigenfunctions $\delta L_{r} /(4 L)$ (dotted line) and $\delta T / T$ (solid line) and of the adiabatic relative temperature variation (dot-dashed line) for the mode $\ell=1, p_{1}$, from the center to the surface of a $10 M_{\odot}$ $\beta$ Cephei model.

non-adiabatic phase difference between the radial and transversal motions, turns out to be always negligible. Comparing Cols. 5 and 6 shows that this approximation is very good for the $\beta$ Cephei model, while it is less accurate for the highest order g-modes of the SPB model. To understand the difference between the two, we recall that $\xi_{h} / \xi_{r} \simeq G M /\left(R^{3} \omega^{2}\right)$ is a result obtained by adopting the Cowling approximation, and assuming that $\delta P / \rho=0$ at the surface. The latter approximation is reasonable if the radiation pressure is negligible at the surface. For hot stars however, this is not the case and it is reflected by a non-negligible contribution of the radiative acceleration in Eq. (20). It is this contribution (the term $a_{\mathrm{R}} \delta T /(\partial T / \partial r)$ to be precise) that makes the difference. Although the equilibrium radiative acceleration $\left(a_{\mathrm{R}}\right)$ is larger for the hotter $\beta$ Cephei stars than for the cooler SPBs, the contribution of the term $a_{\mathrm{R}} \delta T /(\partial T / \partial r)$ is larger for the SPB highest order g-modes than for the $\beta$ Cephei p-modes. This comes from the much larger temperature variations $(\delta T)$ found for the SPB highest order g-modes (for a fixed radial displacement amplitude). Finally, we give in the last column the period of the mode in hours.

As an illustration, we give in Figs. 2 and 3 the amplitudes of the non-adiabatic temperature variation $\left(|\delta T / T|_{\text {nad }}\right)$ and the adiabatic temperature variation $\left(|\delta T / T|_{\mathrm{ad}}=\nabla_{\mathrm{ad}}|\delta P / P|_{\mathrm{ad}}\right)$, from the center to the surface. Figure 2 corresponds to the mode $\ell=1, \mathrm{p}_{1}$ of the $\beta$ Cephei model and Fig. 3 to the mode $\ell=1, \mathrm{~g}_{30}$ of the SPB model. The abscissa corresponds to the logarithm of the equilibrium temperature. We recall that the eigenfunctions are normalized in such a way that $\xi_{r} / R=1$ at $\tau=2 / 3$. A simple comparison between the two graphs shows how different they are in the superficial layers (from the base of the driving region at $\log T \simeq 5.5$ to the surface). It illustrates clearly that adiabatic computations are 


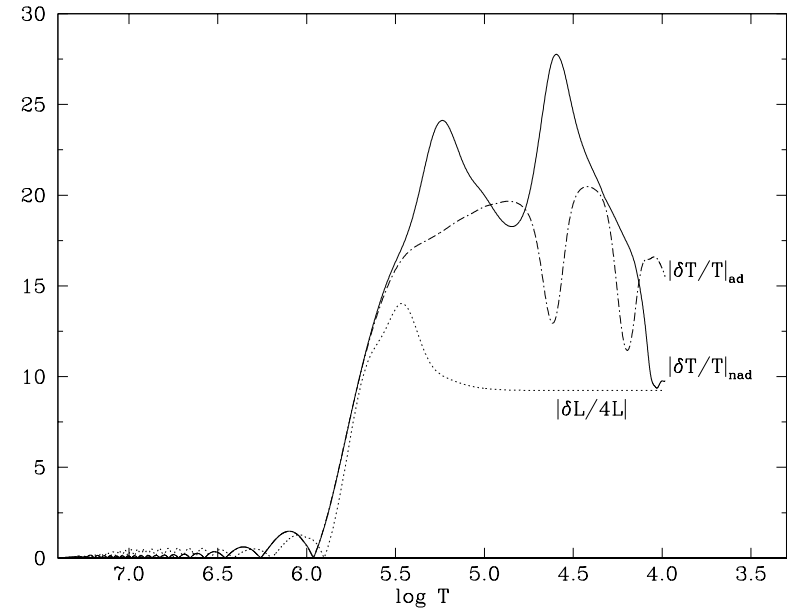

Fig. 3. Amplitude of the non-adiabatic eigenfunctions $\delta L_{r} /(4 L)$ (dotted line) and $\delta T / T$ (solid line) and of the adiabatic relative temperature variation (dot-dashed line) for the mode $\ell=1, g_{30}$, from the center to the surface of a $5 M_{\odot} \mathrm{SPB}$ model.

totally inadequate to obtain reliable values for the temperature variations in the superficial layers of a star.

In the same figures, we also give $\left|\delta L_{r} /(4 L)\right|$. We see that, for the two stars, the graph of $\left|\delta L_{r} /(4 L)\right|$ becomes flat from $\log T \simeq 5$ to the surface. This phenomenon is well known for radial modes and is easily generalized for non-radial modes since for small $\ell$, the terms proportional to $\ell(\ell+1)$ do not play a significant role in Eq. (6). This is due to the very small thermal relaxation time of these layers. Finding already the flat behaviour of $\delta L_{r}$ in much deeper layers than the base of the atmosphere confirms that the hypothesis of radiative equilibrium we adopt in the atmosphere is adequate.

In Figs. 4 and 5, we give the graph of $|\delta T / T|$ in the atmosphere (same models and same modes as previously). The abscissa corresponds to the logarithm of the Rosseland optical depth. The smoothness of the graphs confirms that the two specific treatments in the interior and in the atmosphere match very well at the connecting layer. On the same figures, we give the modulus of the different terms of Eq. (12). For the SPB mode, $\left|\partial \ln T / \partial \ln g_{\mathrm{e}} \delta g_{\mathrm{e}} / g_{\mathrm{e}}\right|$ turns out to be totally negligible and it is not given. For the $\beta$ Cephei model, we see that this quantity remains very small in comparison with the other terms. It is important to note that the term $|\partial \ln T / \partial \ln \tau \delta \tau / \tau|$ is large (particularly for the SPB model). Physically, it means that the Lagrangian variation of the temperature $(\delta T / T)$ and the variation of the temperature at constant optical depth $\left(\Delta_{\tau} T / T \simeq\right.$ $\left.\partial \ln T / \partial \ln T_{\text {eff }} \delta T_{\text {eff }} / T_{\text {eff }}\right)$ are two totally different quantities. It could seem surprising that $|\partial \ln T / \partial \ln \tau \delta \tau / \tau|$ does not go to zero at the outermost layer $(\log \tau=-4.125)$. This comes from the significant values of $\partial \ln T / \partial \ln \tau$ in the Kurucz atmosphere models, even at very small optical depths. In order to examine if this "surprising" phenomenon has a significant impact on the results,

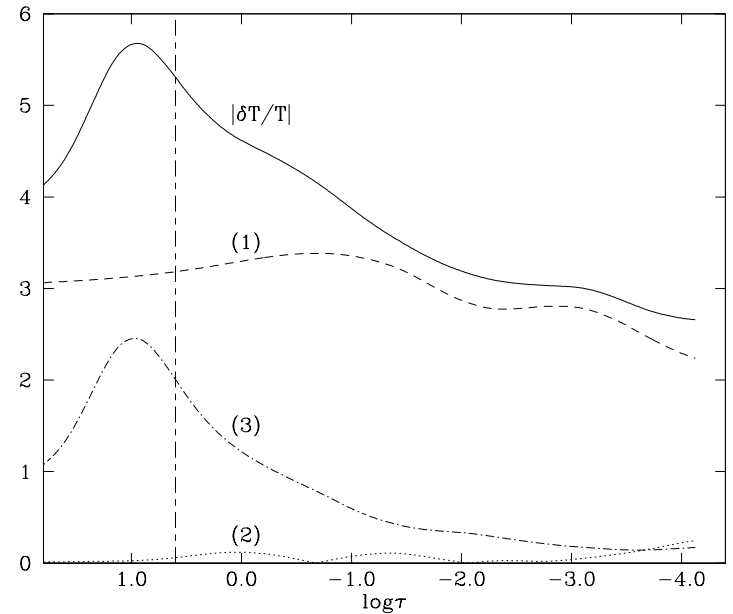

Fig. 4. Amplitude of $\delta T / T$ (solid line) and the moduli of the different contributing terms of the right-hand side of Eq. (12). The dashed line with the label "(1)" is the modulus of the first term ( $\left.\propto \delta T_{\text {eff }} / T_{\text {eff }}\right)$, the dotted line with the label "(2)" is the modulus of the second term $\left(\propto \delta g_{\mathrm{e}} / g_{\mathrm{e}}\right)$, the dot-dashed line with the label "(3)" is the modulus of the third term $(\propto \delta \tau / \tau)$, the vertical line corresponds to the connecting layer between interior and atmosphere. The functions were computed for the mode $\ell=1, \mathrm{p}_{1}$, in the atmosphere of the $10 M_{\odot} \beta$ Cephei model. We note for the comparison that $\left|\delta T_{\text {eff }} / T_{\text {eff }}\right|=3.07$ for this mode.

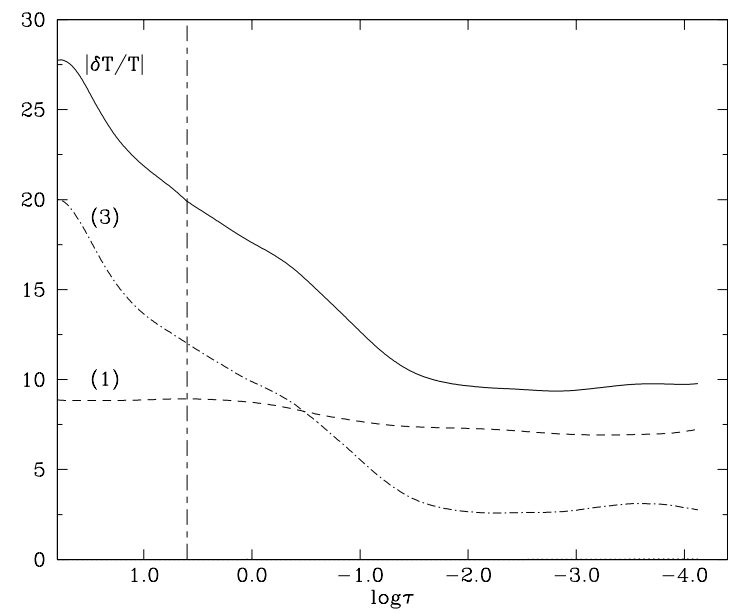

Fig. 5. Same as Fig. 4 for the mode $\ell=1, g_{30}$, in the atmosphere of the $5 M_{\odot}$ SPB model. We note that the contribution of the second term $\left(\propto \delta g_{\mathrm{e}} / g_{\mathrm{e}}\right)$ is so small that it is invisible on this plot, and $\left|\delta T_{\text {eff }} / T_{\text {eff }}\right|=8.80$ for this mode.

we examined the results obtained by putting artificially $\partial \ln T / \partial \ln \tau \delta \tau / \tau$ to zero at the outermost layer (last term of Eq. (28)). Only the values of $\delta T / T$ in the outermost layers (between $\log \tau=-2$ and $\log \tau=-4.125$ ) are significantly affected by this artificial change. The impact on the other results, such as $\delta T_{\text {eff }} / T_{\text {eff }}$ is found to be negligible. We note that $\delta T_{\text {eff }} / T_{\text {eff }}$ will be used instead of $\delta T / T$ in the simulations of line profile time series that will be presented in the second paper of the series.

Comparing the graphs of $|\delta T / T|$ to the values of $\left|\delta T_{\text {eff }} / T_{\text {eff }}\right|$ given in Tables 1 and 2 for the same modes 
shows that they are very different, particularly in the deepest layers of the atmosphere. This leads us to compare the boundary condition we impose on the flux - Eqs. (17) and $(22)$ - to the thermal boundary condition adopted by Dziembowski (1977) and Pesnell (1990), which reads: $\delta L_{r} / L=2 \xi_{r} / r+4 \delta T / T$. For the latter, it is assumed implicitly that $\delta T / T=\delta T_{\text {eff }} / T_{\text {eff }}$ at the outermost layer of the model. Since we found that these two quantities are very different, our treatment appears as a significant improvement.

\section{Conclusions}

In this paper, we derived a method to compute the nonadiabatic eigenfunctions in the interior as well as in the atmosphere of a non-radially pulsating star. Special care was given to the treatment of the perturbed atmosphere, and on this point we improved the method proposed by Dupret (2001). Based on the hypothesis that the atmosphere remains in radiative equilibrium during the pulsation, the non-adiabatic pulsation code we implemented does not use the diffusion approximation in the atmosphere, but assumes instead that the temperature distribution in the local dynamical atmosphere is for each given time and $(\theta, \varphi)$ the same as the one of a Kurucz equilibrium model with varying effective temperature and gravity (Sect. 3.1). Particular care was also taken of the modeling of the radiative acceleration in the momentum equations (Sect. 3.2) and of the determination of the boundary conditions (Sect. 3.3). Finally, some of the results obtained for a typical $\beta$ Cephei star and a typical Slowly Pulsating B star were presented (Sect. 4). On the basis of these results, we can give the following conclusions (to avoid confusion, we recall that all the quantities such as $\delta T / T, \delta T_{\text {eff }} / T_{\text {eff }}$ and $\delta g_{\mathrm{e}} / g_{\mathrm{e}}$ are "local" in the sence that they refer to a given point $(\theta, \varphi)$ of the stellar surface).

- Our non-adiabatic modeling of the temperature variations in the atmosphere based on the hypothesis of radiative equilibrium (Sect. 3.1) permits to find more accurate values of $\delta T / T$ and $\delta T_{\text {eff }} / T_{\text {eff }}$ in the atmosphere than by using the diffusion approximation.

- For a fixed radial displacement amplitude, the variation of the local effective temperature $\left(\delta T_{\text {eff }} / T_{\text {eff }}\right)$ is relatively small compared to the other eigenfunctions for the $\beta$ Cephei $\mathrm{p}$-modes, but it becomes important for the highest order g-modes of the SPB model.

- For the $\beta$ Cephei $\mathrm{p}$-modes and for a fixed radial displacement amplitude, the variation of the gravity from the point of view of a comoving frame $\left(\delta g_{\mathrm{e}} / g_{\mathrm{e}}\right)$ increases rapidly with the radial order of the mode and can go up to significantly large values.

- The Lagrangian variation of the temperature $(\delta T / T)$ decreases a lot from the base to the outermost layer of the atmosphere and is different from the variation of the the local effective temperature
( $\left.\delta T_{\text {eff }} / T_{\text {eff }}\right)$, and from the variation of the temperature at constant optical depth, it is therefore important to make a clear distinction between these quantities.

By using our method, the local $T_{\text {eff }}$ and $\log (g)$ variations come forward in a natural way which is very handy to use in order to make simulations of line profile variations based on Kurucz intensity grids. A method using our nonadiabatic results to compute better line profile time series will be presented in the second paper (De Ridder et al. 2002) of the series.

Acknowledgements. Part of this work was supported by the F.R.I.A. (Fonds pour la formation à la Recherche dans l'Industrie et dans l'Agriculture). The authors are members of the Belgian Asteroseismology Group (B.A.G.).

\section{References}

Aerts, C. 1996, A\&A, 314, 115

Balmforth, N. J. 1992, MNRAS, 255, 603

Buta, R., \& Smith, M. A. 1979, ApJ, 232, 213

Cox, J. P. 1980, Theory of Stellar Pulsation (Princeton Univ. Press, Princeton)

Cugier, H., Dziembowski, W., \& Pamyatnykh, A. 1994, A\&A, 291,143

De Ridder, J., Dupret, M.-A., Neuforge C., \& Aerts, C. 2002, A\&A, 385, 572

Dupret, M. A. 2001, A\&A, 366, 166

Dziembowski, W. 1977, Acta Astron., 27, 95

Gabriel, M. 1989, A\&A, 208, 122

Garrido, R. 2000, in The 6th Vienna Workshop on $\delta$ Scuti and related stars, ed. M. Montgommery, \& M. Breger, PASP Conf. Ser., 210, 67

Heynderickx, D., Waelkens, C., \& Smeyers, P. 1994, A\&AS, 105,447

Kurucz, R. L. 1970, ATLAS: A computer program for calculating model stellar atmospheres, Smithsonian Astrophysical Observatory, special report 309

Kurucz, R. L. 1993, ATLAS9 Stellar Atmosphere programs and $2 \mathrm{~km} / \mathrm{s}^{-1}$ grids. Kurucz CDROM No. 13

Ledoux, P., \& Walraven, Th. 1958, Handbuch der Physik, ed. S. Flügge, 51, 353

Lee, U., Jeffery, C. S., \& Saio, H. 1992, MNRAS, 254, 185

Mihalas, D. 1978, Stellar Atmospheres 2nd ed. (W. H. Freeman and company, San Francisco)

Mihalas, D., \& Weibel-Mihalas, B. 1999, Foundations of Radiation Hydrodynamics (Dover Publications, Inc. Mineola, New York)

Pesnell, W. D. 1990, ApJ, 363, 227

Saio, H., \& Cox, J. P. 1980, ApJ, 236, 549

Telting, J. H., \& Schrijvers, C. 1997, A\&A, 317, 723

Townsend, R. 1997, MNRAS, 284, 839

Unno, W., Osaki, Y., Ando, H., Saio, H., \& Shibahashi, H. 1989, Nonradial oscillations of stars, 2nd ed. (Univ. Tokyo Press, Tokyo)

Watson, R. D. 1988, Ap\&SS, 140, 255 